\title{
Source Memory, Aging and Culture
}

\author{
Hannah Faye Chua ${ }^{a}$ Wenfeng Chen ${ }^{b}$ Denise C. Park ${ }^{c}$ \\ a University of Michigan, Ann Arbor, Mich., USA; ${ }^{b}$ Chinese Academy of Sciences, Beijing, China; \\ 'University of Illinois, Urbana-Champaign, Urbana, III., USA
}

\section{Key Words}

Aging $\cdot$ Culture $\cdot$ Source memory $\cdot$ Cognition $\cdot$ Binding

\begin{abstract}
Background: The present study investigates the possibility that culture affects age differences in context memory. There is evidence that East-Asians process scenes more holistically and show better context memory than Americans. Objective: We examined evidence for differences in binding source to context in young and old Americans and native Chinese. We hypothesized that age effects on source memory could be mitigated due to these cultural differences in processing style. Methods: During incidental encoding, younger and older Chinese and Americans watched a video with statements spoken by four distinct speakers. After a brief interval, participants identified source (experiment 1) or item and source (experiment 2). Results: We observed substantial age-related deficits in source memory in both cultures but little evidence for cultural differences in source or item memory. Conclusion: Basic source memory processes operate similarly across culture and age. The source of holistic processing differences observed between cultures may occur in cognitive operations that are more highly bound to a social context.

Copyright $\odot 2006$ S. Karger AG, Basel
\end{abstract}

In the present study, we consider the possibility that basic cognitive operations that decline with age are moderated by cultural context. There is compelling evidence that with age, a number of fundamental mechanisms de-
(C) 2006 S. Karger AG, Basel

0304-324X/06/0525-0306\$23.50/0

Fax +4161306 1234

E-Mail karger@karger.ch

www.karger.com
Accessible online at:

www.karger.com/ger cline, including speed of processing, working memory function, and binding operations. Nearly all of the data collected to date, however, have relied on Western samples including individuals in European and American countries, as well as Australia. Relatively few data exist describing the cognitive aging of East-Asians (e.g. Chinese, Koreans, Japanese), and the assumption has generally been that the decline of these basic mechanisms with age is a cultural universal [1]. The data that do exist indicate that speed of processing and working memory decline similarly in Western and East-Asian samples [2, 3], and also that speed and working memory are cognitive constructs that are organized similarly across cultures [2]. The study of cross-cultural differences represents an important methodology for understanding the aging process. Whereas chronological age is an indicator of the operation of biological processes over time, culture represents the unique effects of experience on lifespan samples $[1,4]$. To the extent that cognitive aging effects are invariant across cultures, one can make a strong case that age-related declines are biologically-based rather than a result of experience.

There have been many studies conducted on the ability of older adults in Western cultures to bind target items or events to the context in which they occurred. The data suggest that there is substantial evidence that with age, binding operations decline [5]. Older adults show decreased memory for many contextual aspects in which stimuli occur, including the voice or gender of a speaker $[6,7]$, the location or color of a studied target $[8,9]$, whether items were internally or externally generated $[10]$, and whether an item was studied or read $[11,12]$. Not 
only do older adults have difficulty in discriminating among similar features associated with target information [6] which results in a decrement, they also show a decreased ability to bind contextual information to item information to form complex and distinctive memories [13].

To date, there are no studies that examine binding operations in old and young adults across cultures. Binding operations might differ between cultures because there is considerable evidence that sensitivity to contextual information differs between East-Asian and Western cultures [14-16]. Nisbett [14] argues that individuals in East Asian cultures, due to their immersion in an interdependent, group-focused culture, process contextual information more holistically than Westerners. That is, East Asians pay more attention to contextual information. In contrast, Westerners are immersed in an individualistic culture and this results in a more individualistic and analytic, feature-based processing style with less attention to contextual information. In support of the hypothesis that Asians' perceptual operations are more affected by context, Kitayama et al. [16] reported that Japanese adults were more influenced by a contextual frame in judging the length of a line than Americans were. Similarly, Masuda and Nisbett [17] reported that Japanese recalled more background details about a complex scene than did Americans, and also that memory for scenes was more disrupted by contextual changes in Japanese than in Americans. This increased sensitivity to context on the part of East-Asians suggests that members of this culture may be superior to Americans in binding operations, that is, in connecting target to context at encoding.

In the present study, we assessed the effects of culture on binding operations in young and older adults, utilizing stimuli where the target/context relationships were entirely arbitrary. This allowed us to assess binding operations at a fundamental level to determine whether cultural experiences sculpt this cognitive function more efficiently in one culture compared to another. We developed our hypotheses within the biocultural framework developed by Park et al. [1] for understanding the interaction among age, culture, and cognition. Park et al. [1] argued that differences in cognition apparent in young adults as a function of culture may decrease over time on tasks that require effortful and controlled processing, due to neurobiological decline in frontal function. In contrast, culture effects might increase for automatic processes across the lifespan, due to increased reliance with age on these automatic systems and their stability with age. One might expect that the basis for any heightened source memory observed in Chinese would result from holistic processing that causes spontaneous and automatic encoding of contextual information. We expected both American and Chinese older adults to show age-related declines in context (source) memory. However, if young Asians showed superior abilities in binding relative to young Americans, we expected that Chinese older adults would not decline in memory for context as steeply as older Americans. If the bias to process contextual information is an automatic, culturally-biased process for Asians, but not Americans, differences between cultures will get larger with age because automatic processes are resistant to aging whereas effortful processes show pronounce decline with age [1].

In experiment 1 , we compared young and older American and Chinese adults' ability to bind target to source. In this first experiment, we measured binding uncontaminated by item memory recall. At encoding, participants made incidental judgments about the familiarity value of cross-culturally normed facts that were presented aloud by four different speakers. During retrieval, participants saw each fact and were asked to remember the speaker who had presented the fact earlier. In experiment 2 , we increased task difficulty by testing memory for target items as well as their sources so that we could assess cultural differences in binding for items where the target was remembered.

\section{Experiment 1}

\section{Method}

\section{Participants}

Fifty-seven younger adults (29 Americans and 28 Chinese) and 55 older adults (27 Americans and 28 Chinese) participated in this experiment. The younger American adults were undergraduate students (15 male, 14 female) attending the University of Michigan. Older American adults were community dwelling residents (13 male, 14 female) of Washtenaw County, Michigan. Younger Chinese adults were undergraduate students (14 male, 14 female) attending universities in Beijing, and older Chinese adults were community dwelling residents ( 13 male, 15 female) in Beijing, China. All participants received payment for their participation in the experiment.

Details about the sample are presented in table 1. In general, there was more difference in the sample between ages than between cultures. American old participants were on the average slightly older than old Chinese participants, $F(1,109)=29.29, \mathrm{p}<$ 0.001 . Older adult participants completed more years of formal education than younger adults, $F(1,99)=37.56, \mathrm{p}=<0.001$. All participants rated their general health as being slightly better than average (greater than 3 on a 5 -point scale). 
Table 1. Demographic profiles of younger and older American and Chinese groups

\begin{tabular}{|c|c|c|c|c|c|c|c|c|c|}
\hline \multirow[t]{2}{*}{ Culture } & \multirow[t]{2}{*}{ Age group } & \multicolumn{2}{|c|}{ Age, years } & \multicolumn{2}{|c|}{ Education, years } & \multicolumn{2}{|c|}{ Health status ${ }^{\mathrm{a}}$} & \multicolumn{2}{|c|}{ Dot matching } \\
\hline & & mean & $\mathrm{SD}$ & mean & $\mathrm{SD}$ & mean & $\mathrm{SD}$ & mean & $\mathrm{SD}$ \\
\hline \multicolumn{10}{|c|}{ Experiment 1} \\
\hline \multirow[t]{2}{*}{ American } & younger & 20.04 & 1.43 & 14.00 & 1.48 & 3.41 & 0.82 & 13.76 & 3.30 \\
\hline & older & 70.41 & 5.34 & 15.57 & 3.25 & 3.48 & 0.94 & 8.41 & 4.22 \\
\hline \multirow[t]{2}{*}{ Chinese } & younger & 19.07 & 0.78 & 13.00 & 0.93 & 3.43 & 0.69 & 14.89 & 3.41 \\
\hline & older & 65.25 & 1.97 & 16.33 & 1.94 & 3.46 & 0.58 & 8.46 & 3.32 \\
\hline \multicolumn{10}{|c|}{ Experiment 2} \\
\hline \multirow[t]{2}{*}{ American } & younger & 18.84 & 0.80 & 13.77 & 1.07 & 3.81 & 0.75 & 14.35 & 2.77 \\
\hline & older & 67.04 & 4.37 & 14.83 & 3.77 & 3.77 & 0.76 & 12.15 & 4.27 \\
\hline \multirow[t]{2}{*}{ Chinese } & younger & 19.31 & 1.32 & 12.92 & 0.89 & 3.69 & 0.68 & 17.23 & 2.45 \\
\hline & older & 64.32 & 3.27 & 16.15 & 1.54 & 3.54 & 0.64 & 14.86 & 2.85 \\
\hline
\end{tabular}

a Health status was assessed on a 5 -point scale $(1=$ much worse than average; 2 = worse than average; $3=$ average; $4=$ better than average; $5=$ much better than average).

\section{Materials}

\section{Fact Statements}

General fact statements were taken from an encyclopedia, the New York Times 2001 Almanac [18], and the Trivial Pursuit Genus 5 game [19]. They were translated into the Chinese Mandarin language by two bilingual speakers. Only facts where the translations converged were selected. We presented 200 of these statements to a sample (not shown in table 1) of 60 younger and older Chinese and American adults, whose age ranges were similar to participants in our current study. Participants rated how familiar each statement was to them on a five-point scale with endpoints of ' 1 - I definitely do not know this item' to ' 5 - I definitely know this item'. After collecting the familiarity ratings of these 200 statements, we chose 96 statements for inclusion as stimuli in experiments 1 and 2 that had mean familiarity ratings of 2.0 to 4.0 and which did not significantly differ in ratings as a function of culture or the interaction of culture with age group $(p>0.10)$. Examples of statements included are 'Bats are the only mammals capable of flying' and 'The world's most used metal is aluminum'. Half of the statements were randomly assigned for presentation on a videotape, and the other half of the statements were used as distracters for the item recognition test in experiment 2 .

\section{Videos}

Four different versions of a video tape, each containing the same 48 spoken facts, were developed for experiment 1 - two for the Chinese sample and two for the American sample. Within each culture, the two videos differed only in the random order in which the facts were presented. Between cultures, the American and Chinese videos were equivalent in all regards with respect to fact content, order, and pacing, but the speakers and languages were different. The American videos included four European American speakers and the Chinese videos included four Chinese speakers. The speakers represented both ages and gender (young male, young female, old male, old female). The young speakers' ages ranged from 21 to 23 years, and older speakers' ages ranged from 59 to 70 years. Although it would be ideal to have identical faces across the two cultures, after extensive discussion and research, we determined that there was sufficient evidence that individuals have difficulty recognizing different-race faces [20-22]. We decided that using different speakers between cultures, but maintaining cultural mapping of speaker to participant across cultures, better tested the hypotheses of interest.

Each speaker read 12 statements. Speakers and statements on the videos were pseudo-randomly ordered, with the restriction that each speaker would only present two statements successively. There was a seven second interval between statements.

\section{Item and Source Recognition Test}

To test binding of target to context, a questionnaire was developed containing the same 48 acquisition statements reordered with the constraint that no statement from the same speaker was presented more than thrice in succession, and no statements were successive in the original video participants watched. Participants read each statement and determined which speaker presented the statement. The pictures of the four speakers were available on a sheet as reference for the participants.

\section{Procedure}

At acquisition, participants viewed a video in their own language. In order to ensure that participants processed the meaning of the statements, they were told that the experimenters were interested in how familiar the participant was with each statement before he or she heard it in the video. Participants were instructed to rate the familiarity of each of the statements and were not warned that a source memory task would follow.

After viewing the video, participants completed the source memory task to measure binding abilities. Participants were 
asked to read each statement and to try to remember who of the four speakers said the statement. The test was self-paced.

Following the source task, participants also completed the Dot Matching task, which is a speed of processing task similar to the Digits-Symbol task [23]. This task requires participants to compare a dot pattern against key figures and to check 'yes' if the target pattern matches the key and 'no' if it does not. This task was designed to be culturally unbiased for Chinese and American samples.

In addition, participants performed a simple free recall task to assess overall memory ability. Twenty-four pictures from the Snodgrass and Vanderwart [24] picture set were selected as stimuli. These pictures were normed on a large American and Chinese sample and had equal familiarity to both Chinese and American young and older adults [25]. The pictures were presented on slides for five seconds each. Blank slides were shown for one second between the slides. After encoding, participants engaged in a 1-min distractor task, and then were asked to recall the names of the pictures in any order they preferred. The free recall task was used to assess whether particular groups have generally better memory recall abilities than other groups.

\section{Results and Discussion}

Dot Matching Performance and Free Recall of Pictures

These control tasks were used primarily as covariates in the source memory analysis. ANOVA tests with Age and Culture on the variables indicated that younger participants correctly matched more patterns in the Dot Matching task than older adults, $F(1,108)=78.74$, $M S E=12.33, \mathrm{p}<0.001$ (table 1). No other effects were significant. A similar analysis on the free recall data indicated that younger participants reported more names of pictures correctly in the free recall task than older adults $(M s=17.02$ vs. 13.49$), F(1,108)=26.50, M S E=$ $13.32, \mathrm{p}<0.001$. The effect of culture on recall was marginal with Chinese participants remembering more pictures than the Americans, $F(1,108)=3.89, M S E=13.32$, $\mathrm{p}=0.051$. No interaction effect between culture and age was revealed.

\section{Source Memory}

Source memory scores of participants were computed as the proportion of statements correctly attributed to a source divided by the total number of statements studied, which is 48 . Young and old Chinese scored means of 0.67 $(\mathrm{SD}=0.14)$ and $0.43(\mathrm{SD}=0.14)$, respectively, while young and old Americans had means of $0.65(\mathrm{SD}=0.13)$ and $0.43(\mathrm{SD}=0.14)$. A Culture $\times$ Age Group ANOVA on the proportions revealed only a significant main effect of Age. Across the two cultures, younger participants identified proportionately more correct sources for the items than older adults, $F(1,108)=78.31, M S E=0.02, \mathrm{p}<0.001$, mean of $0.66(\mathrm{SD}=0.14)$ for young versus $0.43(\mathrm{SD}=0.14)$ for old. There was no main effect of Culture $(F<1)$ nor was there an interaction effect of Culture and Age $(F<1)$. Covarying source memory scores with participants' familiarity ratings of the statements did not reveal any differences in the patterns of results, nor did covarying source memory scores with participants' free recall scores. (Dot Matching was not used as a covariate since the sample was expected to differ in this measure as a function of age.)

We also conducted an ANOVA to assess whether gender of subject, gender of speaker or age of speaker interacted with the variables of interest. This ANOVA included age of participant, gender of participant, and culture as between group variables; and speaker as a within group variable. Only the Speaker main effect was significant, $F(3,312)=11.56, M S E=0.02, \mathrm{p}<0.001$. This occurred because, for both cultures, performance was worst for the young male speaker. We hypothesized that this was due to the fact that the young male was always presented in the lower right hand corner (in a $2 \times 2$ block of the speakers' faces) on the recognition key (reference guide). In experiment 2, we presented four versions of recognition keys with a different face order, and there was no effect of speaker.

Finally, because we found no evidence of binding differences as a function of culture, we conducted two more analyses to tests alternative hypothesis about binding and source memory. In particular, it might be possible to expect that Chinese and Americans remember different aspects of source. Chinese may have better memory about which group the speakers are associated with, that is gender group or age group, because of the group-focused nature of Chinese culture.

The findings in this experiment are straightforward. Younger adults were better in correctly attributing a statement to its source (speaker) than older adults. No difference was found in source memory scores between the two cultures. The data suggest that the process of binding an arbitrary fact to an arbitrary source is fundamentally the same between the two cultures. Because this experiment focused only on memory for source, it seemed possible that perhaps binding operations might differ between cultures only for items where the target was remembered, and there was no measure of target memory in experiment 1 . To determine if cultural differences might emerge when we examined binding operations only for remembered items, we conducted a second experiment. In experiment 2 , a procedure was used that allowed an assess- 
ment of binding operations only for remembered targets, rather than for every fact. This new experiment addresses the possibility that cultural differences will only emerge when the identity of an item is remembered. If Asians automatically bind and Americans do not, one would expect if an item is correctly recognized, Asians will be more likely than Americans to encode the source with it.

\section{Experiment 2}

A procedure was adopted that commonly has been used to study memory for context $[8,9,26]$. The encoding procedure was similar to experiment 1 , but after the encoding list was presented, participants were given a memory test, which included targets and foils. Participants performed a recognition task of items. When an item was positively identified as a study item, participants recalled its source. If the two cultural groups in each age group performed equally well on item memory tests and have similar levels of source memory, this would confirm the results of experiment 1 , using a more stringent test of binding operations.

\section{Method}

\section{Participants}

52 younger adults (26 Americans and 26 Chinese) and 54 older adults (26 Americans and 28 Chinese) participated in this experiment. The procedure for recruiting the participants was similar to that in study 1 . Young Americans, however, received partial course credit for participation in the experiment. None of the participants in this experiment was also a participant in experiment 1 .

The characteristics of the participants are presented in table 1 and are generally similar to the participants studied in experiment 1. Older American adults were slightly older than older Chinese adults, $F(1,98)=8.24, M S E=7.84, \mathrm{p}=0.005$. Older adults completed more years of formal education, $F(1,98)=26.97, M S E$ $=4.32, \mathrm{p}<0.001$, with older Chinese adults having the highest mean number of years of formal education than the other groups, $F(1,98)=6.92, M S E=4.32, \mathrm{p}=0.01$. Self-reported health status was also comparable among the four groups of participants.

\section{Materials}

The same study and test materials from experiment 1 were used, except for the source recognition test.

Item and Source Recognition Test

The source recognition test was modified to permit an assessment of participants' memories for the items. The item and source identification test consisted of 96 randomized statements, 48 targets and 48 lures. There were four different versions of the reference sheets of speakers, randomly assigned across subjects, so that each speaker appeared in different order in each version of the reference sheets. We note that the same 48 items always served as targets and target/lure identity was not counterbalanced due to the large investment in preparing video tapes with different speakers.

\section{Procedure}

Similar to experiment 1 , participants viewed a video during acquisition. Participants were instructed to rate how familiar the statements presented on the video were, but were not told that they would be given an item and source recognition test later.

In the next $15 \mathrm{~min}$, they completed filler tasks including the Dot Matching task. Each of the participants also randomly received one of the four versions of the reference guide to speakers and a copy of the item and source identification test. Participants were requested to judge whether each of the statements occurred in the video they had just seen or not. If they agreed that the statement was presented in the video, participants were instructed to circle 'Yes' and circle which speaker said the statement. Otherwise, participants should circle 'No'.

Following the item and source identification task, the pictorial free recall task was administered.

\section{Results and Discussion}

\section{Dot Matching Performance and Free Recall}

For the Dot Matching, younger participants correctly matched more patterns than older adults, $F(1,102)=$ $125.28, M S E=6.82, \mathrm{p}<0.001$. Americans overall matched more patterns correctly than the Chinese groups, $F(1$, $102)=8.17, M S E=6.82, \mathrm{p}=0.005$, but the interaction was not significant. On free recall of pictures, there were main effects of age, $F(1,102)=22.35, M S E=11.74, \mathrm{p}<0.001$ and culture, $F(1,102)=8.39, M S E=11.74, \mathrm{p}<0.005$; no interaction of age and culture was found. Younger participants recalled more pictures correctly than the older groups ( $M s=16.65$ vs. 13.56). Chinese participants also recalled more picture items than American participants $(M s=16.0$ vs. 14.11$)$.

\section{Item and Source Memory}

For each participant, hits and false alarms for item recognition were used to calculate a d-prime (d') score for item memory. A $2 \times 2$ ANOVA with Culture and Age as between group variables on the d' values revealed main effects of Age, $F(1,102)=33.07, M S E=0.42, \mathrm{p}<0.001$, and Culture, $F(1,102)=21.78, M S E=0.42, \mathrm{p}<0.001$. As shown in table 2 , younger adults remembered more items than older adults, and Americans remembered more than Chinese. In addition, there was a significant interaction 
Table 2. Means (and SDs) of hit, false alarm, d' and source identification scores for Experiment 2

\begin{tabular}{llllll}
\hline Culture & Age group & Hits & False alarms & d' & $\begin{array}{l}\text { Source } \\
\text { identification }\end{array}$ \\
\hline American & younger & $0.98(0.03)$ & $0.003(0.01)$ & $4.63(0.48)$ & $0.62(0.14)$ \\
& older & $0.94(0.05)$ & $0.005(0.01)$ & $4.22(0.52)$ & $0.39(0.15)$ \\
Chinese & younger & $0.97(0.03)$ & $0.009(0.01)$ & $4.35(0.58)$ & $0.56(0.15)$ \\
& older & $0.89(0.08)$ & $0.05(0.06)$ & $3.31(0.91)$ & $0.40(0.15)$ \\
\hline
\end{tabular}

of Age with Culture, $F(1,102)=6.21, M S E=0.42, \mathrm{p}=$ 0.014 , which occurred because all groups but the old Chinese were close to ceiling in fact recognition. Covarying the d' values with familiarity ratings or free recall scores yielded the same pattern of results produced by the $2 \times$ 2 (Culture $\times$ Age) ANOVA.

Source memory results are presented in table 2 . A source identification score was calculated in the following manner. For items that a participant correctly recognized, a conditional probability for source memory was calculated by dividing the number of correct source responses by the total number of correct item responses. An analysis of variance of the source identification scores yielded a main effect of Age such that younger participants had higher source identification scores than older participants, $F(1,102)=46.76, M S E=0.02, \mathrm{p}<0.001$, but no culture effect $(F<1)$ nor any interaction effect between age and culture. The results did not change after covarying the responses with familiarity ratings or free recall scores of participants. An analysis of the source identification scores that added gender of participant and speaker type yielded no additional significant effects. Finally, two more analyses were conducted that were more liberal in the assessment of correct source identification, as in experiment 1 . When an analysis was conducted on accuracy scores when an item was scored as correct if the correct age group of the speaker was reported, the only significant effect was a main effect of Age, $F(1,102)=$ 58.88, $M S E=23.57, \mathrm{p}<0.001$, that occurred because younger participants performed better than older participants. Similarly, when an item was scored as correct if the correct gender of the speaker was recognized, a main effect of Age also occurred, $F(1,97)=55.80, M S E=23.91$, $\mathrm{p}<0.001$.

Overall, the results suggest that age differences (i.e. younger participants performing better) were consistent on both item and source identification tasks and that there are no culture differences in binding processes. Culture failed to influence participants' performance on the source identification tasks, even when a liberal scor- ing criterion was used. Although the interaction of culture with age was apparent in item memory performance, it was largely due to the slightly worse performance of older Chinese adults, compared to other groups, in correctly identifying studied items. The interaction is not of great concern for three reasons. First, we collected measures of speed of processing and free recall to characterize the sample, and there were only main effects of age, with younger adults performing faster and having better recall than old. Differences between old Americans and Chinese did not differ suggesting that they were equivalent on these measures of basic cognitive resource. Second, although recognition of items was somewhat poorer for the old Chinese, source memory for remembered items did not differ from the old Americans. Finally, the poorer performance of the old Chinese might be interpreted to suggest that they recalled source as well as old Americans, but at the expense of decreased item information. This finding is counter to the hypothesis that older Chinese might be better able to bind target to context than older Americans due to a cultural proclivity to process contextual information. One caveat about the present results is that item memory was near ceiling, although context memory was well below ceiling. It may be the case that on a task where the items were more difficult to remember, context would play an integral role in recalling information and cultural differences might emerge. The data from this study, however, suggest equivalent binding as a function of culture, in accord with the results of experiment 1 , which yielded the same conclusion.

\section{General Discussion}

The study makes two important points. First, there is clear evidence that binding processes operate similarly in cultures that are hypothesized to process context differently. Second, there is evidence that age differences in binding processes are the same across cultures. 
The finding that there were equivalent age differences in source memory in both cultures across two experiments suggests that decreased memory of context is characteristic of cognitive aging across cultures and may represent a fundamental neurobiological signature of cognitive aging. It seems important to consider the implications of this finding by conducting future research that has, on one the hand, greater specificity, and on the other hand, more breadth. With respect to breadth, it will become important to know at what level of analysis age or culture trajectories appear. It may be the case that each culture will prove to be more facilitated if binding tasks are biased toward the culture's views of the individual. For example, given the Western emphasis on individualism, one might expect that if subjects were required to judge intelligence, effects similar to that observed by Rahhal et al. [27], where judgment of character improved source memory in older adults, would occur for Americans but not Asians. Similarly, older Americans might not be facilitated by judging how much 'face' or status a speaker had, as this is a less salient dimension in Western compared to Eastern cultures. Understanding the breadth and depth of culture effects will be important in determining which aspects of cognitive aging are fundamental and which are malleable.

In terms of becoming more specific about mechanisms underlying cultural differences, neuroimaging research can reveal similarities and differences in neural mechanisms that underlie cognitive performance across cultures, as suggested by Park and Gutchess [4]. Recently, Gutchess et al. [28], in an fMRI study, reported that young Americans showed more engagement of object processing areas (middle temporal gyrus and superior parietal gyrus) when judging complex scenes, compared to young East Asians. This finding support arguments by Masuda and Nisbett [17] that East Asians are more sensitive to contextual information than Americans. At the same time, the present data suggest that young and old adults showed equivalent sensitivity to context. The difference between the present findings and those of Masuda and Nisbett [17] and Gutchess et al. [28] is that in the latter studies, the stimuli always involved a meaningful object presented against a complex background scene, so that the target was intimately bound to the context. In the present study, arbitrary speakers were bound to arbitrary facts, so there was no meaningful context for the performance of the binding operation and the same context was used for many items. It may be the case that when a complex context is stripped away from stimuli, binding operations are very similar across cultures. The present work certainly suggests that this is the case, although studies that systematically manipulate these variables are needed.

It is worthwhile to comment, as well, on some of the methodological issues associated with cultural work on aging, as more researchers are likely to direct their attention to this issue [29]. First, sampling is immensely challenging and it is worthwhile to carefully characterize the sample along multiple dimensions. Second, even beyond sampling, the design of culturally sensitive and equivalent stimuli is not easy [see 25, 30 for normed stimuli]. In the present study, it was a difficult decision as to whether to try and present stimuli that used speakers of multiple races that were superficially equivalent, or to use culturally invariant speakers that differed across cultures. We opted to use different speakers between cultures because we believed that the image of Westerners speaking Chinese would be much more novel to Chinese subjects than Chinese speaking English would be to American participants. Fortunately, the present results are interpretable (equivalent age differences within cultures), but other patterns of results would have been more problematic. There is a greater amount of risk inherent in cross-cultural research results being uninterpretable compared to within-culture research on aging. Nevertheless, we believe that a broader and more accurate view of cognitive aging will only be obtained if research that departs from standard Western cognitive aging samples is conducted.

In closing, the present work suggests that the neurobiology of aging takes precedence over culture and that poor source memory may indeed be a universal characteristic of aging, as suggested by Johnson et al. [6] and Hashtroudi et al. [26].

\section{Acknowledgements}

Hannah Faye Chua held an International Fellowship from the American Association of University Women Educational Foundation for years 2002-2003. This research was supported by grant R01-AG15047 from the National Institute on Aging Behavioral Research Program to Denise C. Park. We thank Richard Nisbett, Trey Hedden, Shulan Jiao, Qicheng Jing, Carolyn Yoon, Aysecan Boduroglu, Angela Hall-Gutchess, Kimin Hong, and Yuchen Liu for their assistance. 


\section{References}

1 Park D, Nisbett RE, Hedden T: Aging, culture, and cognition. J Gerontol [B] 1999;54B: P75-P84.

- 2 Hedden T, Park DC, Nisbett R, Ji LJ, Jing Q, Jiao S: Cultural variation in verbal versus spatial neuropsychological function across the life span. Neuropsychology $2002 ; 16: 65-$ 73.

-3 Geary DC, Salthouse TA, Chen G, Fan L: Are East Asian versus American differences in arithmetical ability a recent phenomenon? Dev Psychol 1995;32:254-262.

4 Park DC, Gutchess AH: Aging, cognition, and culture: a neuroscience perspective. Neurosci Biobehav Rev 2003;26:859-867.

$\checkmark 5$ Spencer WD, Raz N: Differential effects of aging on memory for content and context: A meta-analysis. Psychol Aging 1995;10:527539.

6 Johnson MK, De Leonardis DM, Hashtroudi $S$, Ferguson S: Aging and single versues multiple cues in source monitoring. Psychol Aging 1995;10:507-517.

7 Kausler DH, Puckett JM: Adult age differences in memory for sex of voice. Journal of Gerontology 1981;36:44-5016.

8 Park DC, Puglisi JT, Lutz R: Spatial memory in older adults: effects of intentionality. J Gerontol 1982;37:330-335.

9 Park DC, Puglisi JT: Older adults' memory for the color of pictures and words. J Gerontol 1985;40:198-204.

10 Rabinowitz JC: Judgments of origin and generation effects: comparison between young and elderly adults. Psychol Aging 1989;4: 259-268.

11 Hedden T, Park DC: Aging and interference in verbal working memory. Psychol Aging 2001;16:666-681.
12 Hedden T, Park DC: Contributions of source and inhibitory mechanisms to age-related retroactive interference in verbal working memory. J Exp Psychol. In press.

13 Chalfonte BL, Johnson MK: Feature memory and binding in young and older adults. Memory Cogn 1996;24:403-416.

14 Nisbett R: The Geography of Thought: How Asians and Westerners Think Differently ... and Why. New York, The Free Press, 2003.

15 Nisbett RE, Peng K, Choi I, Norenzayan A: Culture and systems of thought: holistic vs. analytic cognition. Psychol Rev 2001;108: 291- 310.

16 Kitayama S, Duffy S, Kawamura T, Larsen JT: Perceiving an object and its context in different cultures: a cultural look at New Look. Psychol Sci 2003;14:201-206.

17 Masuda T, Nisbett R: Attending holistically versus analytically: comparing the context sensitivity of Japanese and Americans. J Pers Soc Psychol 2001;81:922-934.

18 Wright J (ed): The New York Times 2001 almanac. USA, Penguin Group, 2000.

19 Horn Abbott Ltd: Trivial pursuit genus 5. USA, Author, 2000.

20 Levin DT: Race as a visual feature: using visual search and perceptual discrimination tasks to understand face categories and the cross-race recognition deficit. J Exp Psychol 2000;129:559-574.

-21 Maclin O, Malpass R: Racial categorization of faces: the ambiguous race face effect. Psychol Publ Policy Law 2001;7:98-118.

22 Sporor S: Recognizing faces of other ethnic groups: an integration of theories. Psychol Publ Policy Law 2001;7:36-97.
23 Wechsler D: Wechsler Adult Intelligence Scale - Revised. San Antonio, Psychological Corporation, 1981.

24 Snodgrass J, Vanderwart M: A standardized set of 260 pictures: norms and name agreement, image agreement, familiarity, and visual complexity. J Exp Psychol 1980;6:174215 .

25 Yoon C, Luo T, Feinberg F, Hedden T, Gutchess AH, Chen HY, Mikels JA, Jiao S, Park DC: A cross-culturally standardized set of pictures for younger and older adults: American and Chinese norms for name agreement, concept agreement and familiarity. Submitted, 2002.

26 Hashtroudi S, Johnson MK, Chrosniak LD: Aging and source monitoring. Psychol Aging 1989;4:106-112.

27 Rahhal TA, May CP, Hasher L: Truth and character: sources that older adults can remember. Psychol Sci 2002;13:101-105.

28 Gutchess A, Welsh R, Boduraglu A, Park DC: Cultural differences iin neural function associated with object processing. Cogn Affect Behav Neurosci, in press.

29 Hedden T, Park DC: Culture, aging, and cognitive aspects of communication; in Charness N, Park DC, Sabel B (eds): Communication, Technology, and Aging: Opportunities and Challenges for the Future. New York, Springer, 2001, pp 81-108.

30 Yoon C, Feinberg F, Hu P, Gutchess AH, Hedden T, Chen HY, Jing Q, Cui Y, Park DC: Category norms as a function of culture and age: comparisons of item response to 105 categories by American and Chinese adults. Submitted, 2002 